\title{
Research on Key Techniques of Digital Signal Processing Theory for Electric Machines and Motor Control
}

\author{
ZENG Song ${ }^{1, a}$ \\ ${ }^{1}$ ChangJiang Institute Of Technology, WuHan 430212, China \\ a ethree_blue@163.com
}

Keywords: Digital Signal Processing; Electric Machines; Motor Control; Crucial Technology

\begin{abstract}
With the fast and rapid advancement in computer and control technology, it is essential and necessary for the electronic products to be elaborated and sophisticated, and the internal functions are various and high speed. In the late 1990s, the emergence of the new type permanent magnet mechanism and electronic operation for medium voltage synchronization technology in the field of implementation provides a hardware foundation. In order to satisfy the high reliability of vacuum circuit breaker operation, our country is in-depth study and gradually promote and matching the electronic control system of permanent magnetic actuator. In this paper, we conduct research on key techniques of digital signal processing theory for electric machines and motor control. The experimental result shows the effectiveness of the proposed method.
\end{abstract}

\section{Introduction}

With the fast and rapid advancement in computer and control technology, it is essential and necessary for the electronic products to be elaborated and sophisticated, and the internal functions are various and high speed. Synchronization (or phase) operations are electrical appliances intelligent frontier technology, this technology can effectively weaken the breaker points when closing the inrush current, over voltage, such as transient impact, has been widely used and good results have been achieved. Domestic already in theory and practice of synchronization technology has carried on the beneficial attempt, but the applications of the synchronization technology in the field of medium voltage applications haven't seen a report [1-2]. A key to realize synchronous circuit breaker operation technology is its action time dispersion cannot be more than $1 \mathrm{~ms}$, otherwise the technology compared with other methods, there is no obvious advantage, and such a demand for the traditional spring mechanism and electromagnetic mechanism is difficult to achieve. In the late 1990s, the emergence of the new type permanent magnet mechanism and electronic operation for medium voltage synchronization technology in the field of implementation provides a hardware foundation. In order to satisfy the high reliability of vacuum circuit breaker operation, our country is in-depth study and gradually promote and matching the electronic control system of permanent magnetic actuator. At present was still in the stage of research and development of permanent magnetic actuator, can only do small and medium capacity level [3-5]. Magnetic system and the coil excitation mode is the output of the permanent magnetic actuator load can be a good match with the vacuum circuit breaker mechanical characteristic of the key factors. I know most for permanent magnetic actuator controller is given priority to with protection, measurement, and other functions, to be implemented according to the specific load synchronous operation.

A common problem with sensor-less motor drive system is its starting performance. The sensor-less algorithm using above, measured signal is too small to make accurate location estimation in static or low speed motor. The most common solution to the problem is the open loop start method from a predetermined rotor position. The inductive magnetic field of the permanent magnet rotor will align direction corresponding [6]. With a known initial rotor position, an open-loop control application increased the frequency of the injection current speed motor from stagnation. However, especially three-phase winding three-phase induction motor, thus cause the stator magnetic field are two opposite directions. With the rapid development of integrated circuit, the digital motor control 
system has been widely used software implementation based on microcontrollers and digital signal processor (DSP) [7-8]. These methods provide flexibility and are suitable for motor drive applications.

In this paper, we conduct research on key techniques of digital signal processing theory for electric machines and motor control. The proposed control schemes have been implemented on a single-chip DSP controller (TMS320LF2407A) to verify the performance and feasibility for three phase motors. The effectiveness and feasibility of our system is proven in the experiment part.

\section{Our Proposed Methodology}

The Modelling of the Electric Motors. Induction motor and an appropriate power converters and the speed or position feedback, in many complex control applications have become popular. The contestant design and compensation system requires a precise mathematical model and all of the components of the system. Permanent magnet mechanism controlled by power electronic components storage capacitor discharge of excitation coil, complete the closing operation, and it is implemented in a permanent magnet lock, is the perfect combination of electromagnetic system and permanent magnet system; and the force-trip characteristics and the requirements of the vacuum circuit breaker is very close. It significantly reduced the transmission links, improve the reaction speed, precision and the reliability of operation, its more far-reaching significance is greatly improving the controllability, decentralized control time from the original mechanical system of millisecond progress into electrical signal control microsecond, from mechanical energy storage, mechanical tripping progress to electric energy storage, electronic tripping signals directly trigger action. This study a simple modeling method is proposed to meet the characteristics of the three-phase induction motor, and discusses the static and dynamic drive circuit and the relationship between the actual motor. The model is shown in the figure 1 . The descriptive equation is shown in the formula 1-3.

$$
\begin{aligned}
& v=L_{s} \frac{d i}{d t}+i R_{s}+v_{e m f} \\
& T_{e}=J_{m} \frac{d w_{r}}{d t}+B_{m} w_{r}+T_{L} \\
& i(t)=\frac{v-v_{e m f}}{R_{s}}\left(1-e^{\left(-R_{s} t / L_{s}\right)}\right)
\end{aligned}
$$

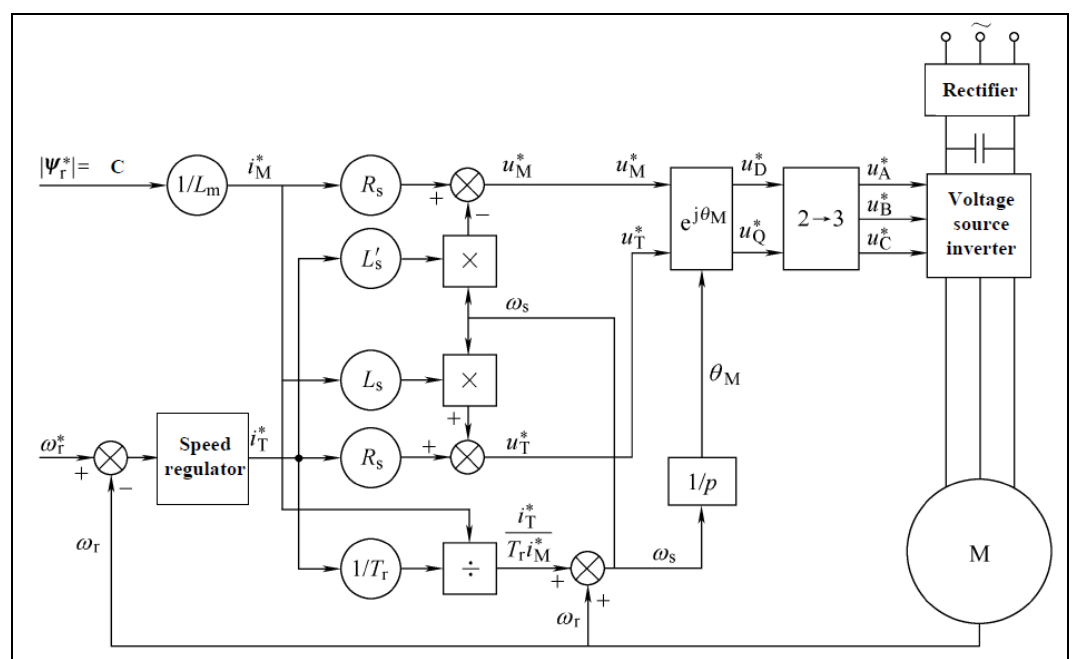

Fig.1 The Schematic of the Full-bridge Inverter for Our System

Typical Analog to Digital Conversion. Power converters incorporate current and/or voltage feedback, requiring analog inputs. The essence of the synchronization technology control circuit breaker is in the desired phase close or open circuit voltage or current. Based on the reactive power 
compensation in non-ground neutral capacitor is star group as an example, the analysis on the reference voltage zero synchronous switching control method is introduced. Sampled data are passed to CPU through interrupts or direct memory access operations implemented through memory sharing mechanisms. The latter eliminates overheads and latencies, typical of interrupts. In the figure 2, we draw the organizational structure of conversion.

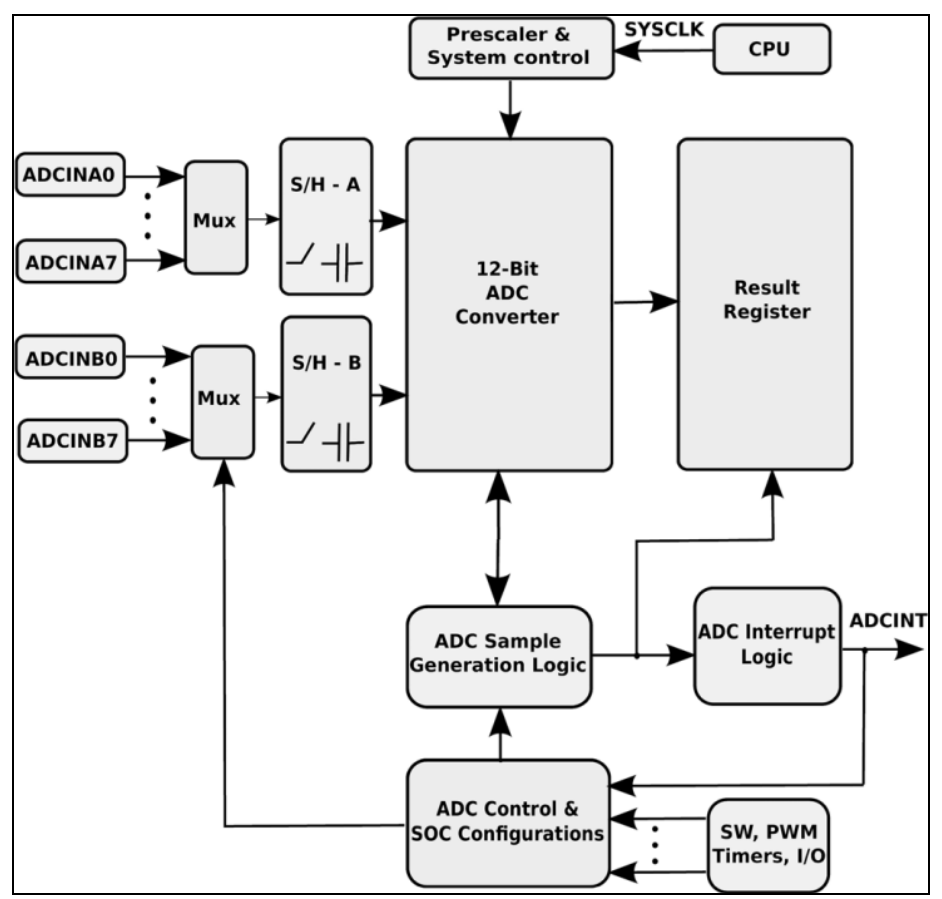

Fig.2The Organizational Structure of Conversion

Optimization of Three Phase Induction Motors. Due to the machine design and structure, the induced back-EMF in the three phase induction motors is highly nonlinear and contains harmonics. Known from the analysis of the above, the reference zero point detection accuracy is vital for synchronous control. Main program set in the remote control instructions (including brake, closing, lock and unlock), contact in place, the time has come regularly, external interrupt flag register, loss of pressure, flow, the control voltage limit, such as circuit breaker contact impending fault sign the register. System self-check and initialization, first determine the system normal again after detection control voltage, ambient temperature environment parameters, such as, system voltage, current, and then track the status of the above work, failure marks register, such as setting flags have been found in the corresponding processing subroutine. The commonly used hardware zero comparator method by the comparator offset voltage, the influence of high harmonics and white noise is larger. In order to overcome these defects, usable digital filtering method to detect the zero. Target phase closing contact the three-phase minimum delay is shown in the formula 4.

$$
\left\{\begin{array}{l}
t_{d A}=1 / 2 f-\left[t_{c 1 A} \bmod (1 / 2 f)\right]+10.0 \\
t_{d B}=1 / 2 f-\left[t_{c 1 B} \bmod (1 / 2 f)\right]+8.33 \\
t_{d C}=1 / 2 f-\left[t_{c 1 C} \bmod (1 / 2 f)\right]+5.00
\end{array}\right.
$$

\section{Experiment Analysis and Simulation}

The current-loop sensor-less control scheme can be verified by computer simulation with proposed model. This research adopts the digital PI controller to realize the current regulation, to ensure accurate measurement of stator current tracking the required value, shorten transient intervals as much as possible. For the sake of simplicity, we adopt the digital design method it determines the equivalent continuous time model of the whole system, using it in a continuous time stable controller design of the feedback loop, in the end, the continuous time controller is converted into an equivalent 
discrete time. The use of digital design advantage of some well-known controller design method for the continuous time model development is analyzed. In the figure 3 we show the simulation result.

For entire speed control range, both of RMS value and peak value is lower when using closed-loop current-mode control. That is to say, the closed-loop current-mode control of sensor-less motor drive is a low cost and high efficiency solution for three phase induction motors.

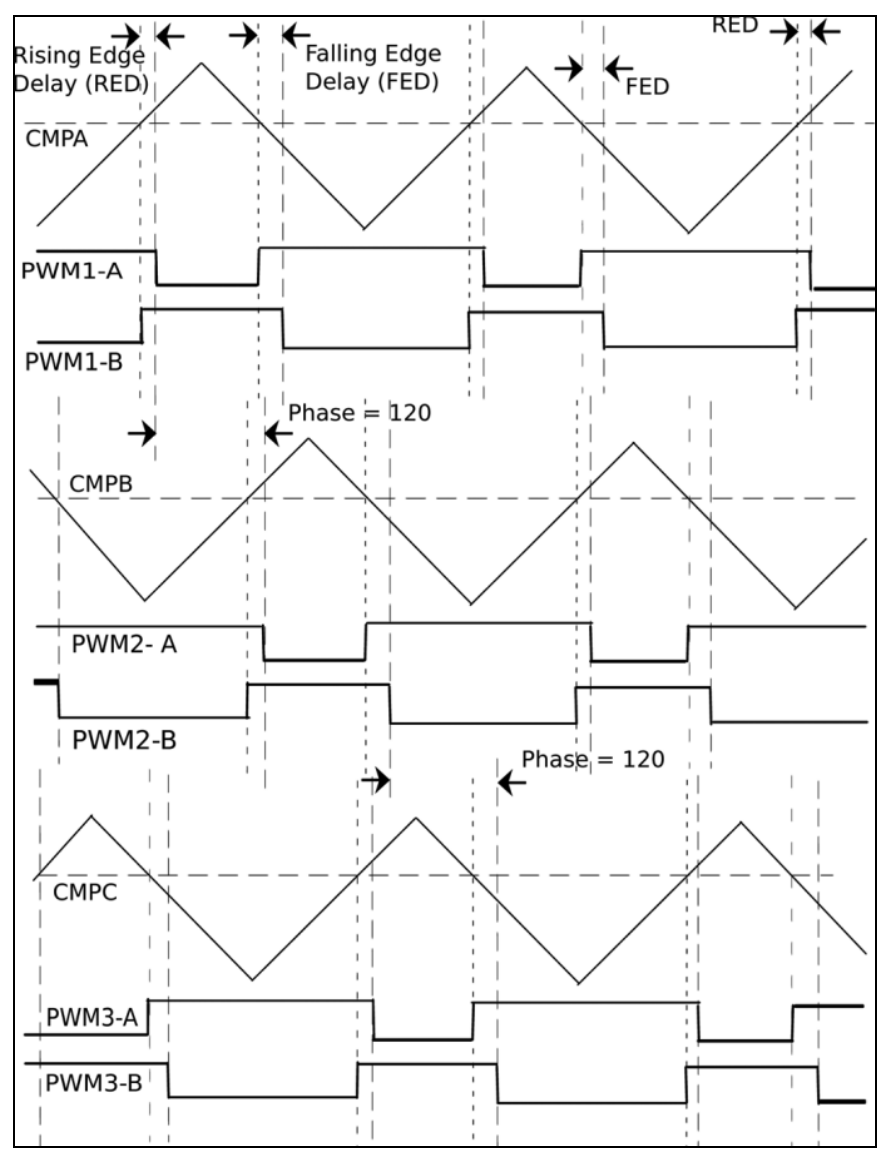

Fig.4The Simulation of Our Research

\section{Conclusion and Summary}

With the fast and rapid advancement in computer and control technology, it is essential and necessary for the electronic products to be elaborated and sophisticated, and the internal functions are various and high speed. In the late 1990s, the emergence of the new type permanent magnet mechanism and electronic operation for medium voltage synchronization technology in the field of implementation provides a hardware foundation. In order to satisfy the high reliability of vacuum circuit breaker operation, our country is in-depth study and gradually promote and matching the electronic control system of permanent magnetic actuator. At present was still in the stage of research and development of permanent magnetic actuator, can only do small and medium capacity level. In this paper, we conduct research on key techniques of digital signal processing theory for electric machines and motor control. The proposed control schemes have been implemented on a single-chip DSP controller (TMS320LF2407A) to verify the performance and feasibility for three phase motors. The experimental result shows the effectiveness of the proposed method. In the future, we plan to conduct more flexible techniques to deal with the difficulties.

\section{References}

[1] Jia-yong, C., You-ping, C., Qiong-hua, Z., \& Zu-de, Z. (2002). A review of control technology of switched reluctance motor. Electric Machines and Control, 6, 1, 1-80. 
[2] Lu, Z., Sheng, H., Hess, H. L., \& Buck, K. M. (2005). The modeling and simulation of a permanent magnet synchronous motor with direct torque control based on matlab/simulink. Electric Machines and Drives, 2005 IEEE International Conference on, 7 pp. - 1156.

[3] Ping, S., \& Jian-qiu, Y. (2008). Permanent ac servo motor and control system of lockstitch sewing machine. Electric Machines \& Control Application.

[4] Ze-yun, C., Yong, K., He-qing, Z., \& Zhi-xin, X. (2007). Modeling and simulation of asynchronous motor vector control system. Electric Machines \& Control Application.

[5] Gonzalez-Llorente, J., Ortiz-Rivera, E. I., \& Diaz, A. (2009). A maximum power point tracker using positive feedforward control based on the dc motor dynamics and pvm mathematical model. Electric Machines and Drives Conference, 2009. IEMDC '09. IEEE International, 259 - 264.

[6] Blum, D. W. (1998). Dynamo-electric machines and control and operating system for the same. US, US6222331 B1.

[7] Zhi-feng, Z., Ren-yuan, T., Jian-guang, Z., \& Bao-dong, B. (2010). Nonsingular terminal sliding mode control of flux and torque for induction motor. Electric Machines and Control, 14, 12, 47-51.

[8] Zidani, F., Benbouzid, M. E. H., Diallo, D., \& Benchaib, A. (2003). Active fault-tolerant control of induction motor drives in EV and HEV against sensor failures using a fuzzy decision system. Electric Machines and Drives Conference, 2003. IEMDC'03. IEEE International (Vol.2, pp.677 683). IEEE. 